\title{
Independence of the Final Catalyst Activity Profile on the Details of Reactant Admission
}

\author{
D. Constales ${ }^{1}$, G.S. Yablonsky², G.B. Marin ${ }^{3}$ \\ ${ }^{1}$ Department of Mathematical Analysis, Ghent University \\ Krijgslaan 281 S-22, 9000 Gent (Belgium) \\ ${ }^{2}$ Parks College of Engineering, Aviation and Technology, Saint Louis University \\ Saint Louis, MO63103 (USA) \\ ${ }^{3}$ Laboratory for Chemical Technology, Ghent University \\ Technology Park Zwijnaarde 914, 9052 Gent (Belgium)
}

\begin{abstract}
It is shown analytically, based on pulse-response experiments, that under special conditions the activity profile of a prepared catalytic system depends only on the total amount of admitted substance. This property, previously found computationally, is here established mathematically for porous and nonporous catalysts in different pulse reactors.
\end{abstract}

Keywords and phrases: catalyst preparation, catalyst activity profile, Temporal Analysis of Products (TAP), pulse-response experiment

Mathematics Subject Classification: 35K57

\section{Introduction}

The optimal or controlled design of catalytic systems at all scales, i.e., active site, catalyst pellet or membrane, and catalytic reactor, remains one of the most important problems in chemical technology, $[1,2,5-8,10]$. Impregnation procedures, one or many-component, wet or dry, can be considered as the main stages of the catalyst preparation technique on a given support. Lee and Aris [5] formulated the basic model equations of the impregnation step for a porous catalyst, assuming the adsorption of the impregnant is the major physicochemical process in determining the distribution of the corresponding active sites. In this "transport-reaction" model, the transport dependence was governed by Fick's diffusion law and the adsorption process was considered to be reversible and monomolecular. Based on this model, a systematic computational study of the active site distribution within the catalytic pellet was performed. Nevertheless, since this pioneering theoretical work on catalyst preparation procedures, until now no rigorous analytical result was obtained that could be considered as the starting point of a theory that would allow to predict the spatial distribution of the catalytic active sites upon impregnation.

Recently, in computational pulse-response adsorption experiments by [9] a very interesting phenomenon was found: the calculated profiles of catalytic active sites were identical if the number of molecules pulsed during the adsorption procedure was the same. Whether ten 'small' pulses containing $10^{13}$ molecules each

\footnotetext{
*Corresponding author. E-mail: denis.constales@ugent.be
} 
or one 'large' pulse of $10^{14}$ molecules are injected, the calculated profiles of sorbates, and hence of the final active sites, are identical. Phanawadee et al. [9] analysed the model of Temporal Analysis of Products (TAP) response gas-solid experiments, see $[3,4]$. The TAP-experiment is performed in the Knudsen diffusion regime, in which the diffusion coefficient does not depend on the chemical mixture composition, nor on the total pressure. In the modeling by Phanawadee et al. [9] it was assumed that the adsorption process is irreversible and monomolecular. Irreversible monomolecular adsorption in a TAP reactor can be considered as the simplest process representing impregnation of supports for catalyst preparation. Our analytical results are obtained specifically for this process; however, the same analysis can be applied to many processes of material preparation with controlled profile of active substances, e.g., preparation of materials via wet impregnation and optimisation of catalyst profile during the deactivation process.

As mentioned, the computational results obtained by [9] are that the profiles of catalytic active sites, in this case the adsorbed active substance, are functions of the total number of pulsed molecules only, not of any other parameters of the pulse procedure, such as the number of pulses and the number of molecules per pulse. This property was found for many different cases studied in the TAP-setup:

- for nonporous and for porous catalysts

- for different configurations of the TAP reactor, i.e., one-zone, thin zone and three-zone

The goal of this paper is to provide a rigorous theoretical explanation of the computational result of [9], and hence also a clue to further development of catalyst design theory.

\section{Single bed zone, nonporous catalyst}

The equations in a single zone nonporous reactor are:

- the diffusion/reaction equation for the gas concentration $C$,

$$
\epsilon_{b} \frac{\partial C(x, t)}{\partial t}=D_{b} \frac{\partial^{2} C(x, t)}{\partial x^{2}}-\rho_{s}\left(1-\epsilon_{b}\right) k C(x, t)(1-\theta(x, t)), \quad 0 \leq x \leq L, 0 \leq t<+\infty
$$

- the reaction equation for the coverage $\theta$ of the catalytic active sites,

$$
\frac{\partial \theta(x, t)}{\partial t}=k C(x, t)(1-\theta(x, t)), \quad 0 \leq x \leq L, \quad 0 \leq t<+\infty .
$$

This equation reflects the irreversible adsorption on the catalyst support, which is the simplest procedure of catalyst preparation.

- The flux boundary condition on $C$ at the reactor inlet (where $x=0$ ),

$$
-D_{b} \frac{\partial C}{\partial x}(0, t)=I(t) \frac{N_{p}}{A}, \quad 0 \leq t<+\infty .
$$

this boundary condition reflects the TAP pulse-response experiment, where $I(t)$ can be any prescribed time evolution of injected flux. In the linear setting, a single pulse $\delta\left(t-0^{+}\right)$or series of pulses are usually considered; it will follow from the present analysis that this distributional limit can also be taken here.

- The boundary condition on the value of $C$ at the reactor outlet (where $x=L$ ).

$$
C(L, t)=0, \quad 0 \leq t<+\infty .
$$

This boundary condition is an approximation of the high vacuum outside the TAP reactor.

- The initial condition on $C$ (empty reactor),

$$
C(x, 0)=0, \quad 0 \leq x \leq L,
$$


- the initial coverage $\theta_{0}$,

$$
\theta(x, 0)=\theta_{0}(x), \quad 0 \leq x \leq L .
$$

The system (2.1)-(2.6) is nonlinear due to the presence of the product $C \theta$ on the right-hand sides of $(2.1)$ and (2.2), reflecting monomolecular adsorption; consequently there is no closed solution in mathematical terms. But the special properties of this system do allow to integrate it from $t=0$ to $+\infty$, as follows. Rewriting (2.2) as

$$
\frac{1}{1-\theta(x, t)} \frac{\partial \theta(x, t)}{\partial t}=k C(x, t), \quad 0 \leq x \leq L, \quad 0 \leq t<+\infty
$$

both sides can be integrated from $t=0$ to $+\infty$, yielding

$$
\ln \frac{1-\theta_{0}(x)}{1-\theta_{+\infty}(x)}=k M(x)
$$

where by $\theta_{+\infty}(x)=\lim _{t \rightarrow \infty} \theta(x, t)$ we denote the final coverage, and by $M(x)=\int_{0}^{+\infty} C(x, t) d t$ the zeroth moment of $C(x, t)$. This $M(x)$ is therefore the total concentration exposure over time, which is known in pharmacokinetics as the AUC, Area Under Concentration. Solving (2.8) for $\theta_{+\infty}(t)$ we find that

$$
\theta_{+\infty}(x)=1-\left(1-\theta_{0}(x)\right) \exp (-k M(x)) .
$$

The expression $k M(x)$, which is dimensionless, characterises the efficiency of exposure, and will be denoted by $\tilde{M}$.

Therefore, in this case the final catalyst composition, i.e. the final concentration of catalytic sites, is uniquely defined by the integral amount of gas substance concentrations related to the given position.

Next, we replace the reaction term using (2.2) to obtain

$$
\epsilon_{b} \frac{\partial C(x, t)}{\partial t}=D_{b} \frac{\partial^{2} C(x, t)}{\partial x^{2}}-\rho_{s}\left(1-\epsilon_{b}\right) \frac{\partial \theta(x, t)}{\partial t},
$$

and integrate over $0 \leq t<+\infty$ to produce

$$
0=D_{b} \frac{d^{2} M(x)}{d x^{2}}-\rho_{s}\left(1-\epsilon_{b}\right)\left(\theta_{+\infty}(x)-\theta_{0}(x)\right)
$$

where the vanishing of the left-hand side is due to $C(x, 0)=C(x,+\infty)=0$ in the whole reactor. Substituting (2.9) into this we obtain a second-order ordinary differential equation,

$$
0=D_{b} \frac{d^{2} M(x)}{d x^{2}}-\rho_{s}\left(1-\epsilon_{b}\right)\left(1-\theta_{0}(x)\right)(1-\exp (-k M(x)))
$$

and integrating the boundary conditions (2.3) and (2.4) leads to

$$
-D_{b} \frac{d M}{d x}(0)=\frac{N_{p}}{A} \int_{0}^{+\infty} I(t) d t
$$

and

$$
M(L)=0 .
$$

which provide boundary conditions on $M$ in eq. (2.12). Equations (2.12)-(2.14) defines the function $M(x)$ uniquely over $0 \leq x \leq L$, and through (2.9) the final coverage $\theta_{+\infty}(x)$ is also uniquely defined.

In $(2.12)-(2.14)$ only the total amount of gas molecules pulsed in, the integral over time of $I(t)$, occurs; consequently the final coverage only depends on this total amount, but not on the detailed time evolution of the injected gas flux $I(t)$. It should be stressed that this is an exact mathematical result, involving no approximations. 
Introducing the dimensionless length coordinate $\xi=x / L$ and the dimensionless concentration moment $\tilde{M}(\xi)=k M(x)$, equation $(2.12)$ can be rewritten as

$$
\frac{d^{2} \tilde{M}(\xi)}{d \xi^{2}}=\operatorname{Da}\left(1-\theta_{0}(\xi)\right)(1-\exp (-\tilde{M}(\xi))), \quad 0 \leq \xi \leq 1,
$$

where $\mathrm{Da}=k \rho_{s}\left(1-\epsilon_{b}\right) L^{2} / D_{b}$ is the Damköhler number. The boundary conditions are then for the inlet $\frac{d \tilde{M}}{d \xi}(0)=-\tilde{p}$ where $\tilde{p}=\frac{N_{p} \mathrm{Da}}{\rho_{s}\left(1-\epsilon_{b}\right) A L} \int_{0}^{+\infty} I(t) d t$ is the dimensionless total amount pulsed in; and for the outlet, $\tilde{M}(1)=0$.

When the initial coverage $\theta_{0}(x)$ is zero, it is possible to integrate (2.15) as follows: let $\tilde{V}$ denote $d \tilde{M} / d \xi$, then $d^{2} \tilde{M} / d \xi^{2}=\tilde{V}(d \tilde{V} / d \tilde{M})$ hence

$$
\tilde{V} \frac{d \tilde{V}}{d \tilde{M}}=\operatorname{Da}(1-\exp (-\tilde{M}))
$$

so that, writing $\tilde{M}_{l}=\tilde{M}(0)$ ( $l$ stands for 'left'), and integrating from $\tilde{M}$ to $\tilde{M}_{l}$,

$$
\frac{1}{2}\left(\tilde{p}^{2}-\tilde{V}^{2}\right)=\operatorname{Da}\left(\tilde{M}_{l}-\tilde{M}+\exp \left(-\tilde{M}_{l}\right)-\exp (-\tilde{M})\right)
$$

which leads to the implicit solution

$$
\int_{\tilde{M}(\xi)}^{\tilde{M}_{l}} \frac{d \tilde{M}}{\sqrt{\tilde{p}^{2}-2 \operatorname{Da}\left(\tilde{M}_{l}-\tilde{M}+\exp \left(-\tilde{M}_{l}\right)-\exp (-\tilde{M})\right)}}=\xi
$$

where $M_{l}$ must satisfy the equation

$$
\int_{0}^{\tilde{M}_{l}} \frac{d \tilde{M}}{\sqrt{\tilde{p}^{2}-2 \operatorname{Da}\left(\tilde{M}_{l}-\tilde{M}+\exp \left(-\tilde{M}_{l}\right)-\exp (-\tilde{M})\right)}}=1 .
$$

The final coverage profile is then given by

$$
\theta_{+\infty}(\xi)=1-\exp (-\tilde{M}(\xi))
$$

In Fig. 1 typical catalyst profiles are shown for different values of Da and $\tilde{p}$. Based on these dependencies we know in advance the final catalyst profile within the single zone if we know the physicochemical parameters of the system (diffusivity $D$, rate constant $k$, dimensionless total amount pulsed in $\tilde{p}$ ).

If $\tilde{M}(\xi)$ is small, this is approximately $\theta_{+\infty}(\xi)=\tilde{M}(\xi)=k M(x)$. This simple equation can be used for the estimation of the catalytic active site profile when its concentration is small enough.

\section{Three-zone reactor, porous catalyst}

If the three zones have lengths $L_{1}, L_{2}$ and $L_{3}$, and the first and third are inert but the second is reactive, the equations in the three-zone nonporous reactor are:

- the diffusion or diffusion/reaction equations for the gas concentration $C$,

$$
\begin{gathered}
\epsilon_{1} \frac{\partial C(x, t)}{\partial t}=D_{1} \frac{\partial^{2} C(x, t)}{\partial x^{2}}, \quad 0 \leq x<L_{1}, \quad 0 \leq t<+\infty, \\
\epsilon_{2} \frac{\partial C(x, t)}{\partial t}=D_{2} \frac{\partial^{2} C(x, t)}{\partial x^{2}}-\lambda\left(1-\epsilon_{2}\right) D_{p} \frac{\partial C^{p}(x, y, t)}{\partial y}(x, R, t), \\
L_{1}<x<L_{1}+L_{2}, \quad 0 \leq t<+\infty,
\end{gathered}
$$




$$
\epsilon_{3} \frac{\partial C(x, t)}{\partial t}=D_{3} \frac{\partial^{2} C(x, t)}{\partial x^{2}}, \quad L_{1}+L_{2}<x<L_{1}+L_{2}+L_{3}, \quad 0 \leq t<+\infty .
$$

where $\lambda$ is the external surface area of the pellet per volume of the pellet, typically $\lambda=3 / R ; C^{p}(x, y, t)$ denotes the concentration in support, the internal variable $y$ measures distance inside the pellet, $0 \leq y \leq R$, and the pellet-level concentration satisfies the $d$-dimensional diffusion-reaction equation, where $d=1,2,3$, for a linear, cylindrical, or spherical pellet respectively.

$$
\begin{aligned}
\epsilon_{p} \frac{\partial C^{p}(x, y, t)}{\partial t}= & D_{p}\left(\frac{\partial^{2} C^{p}(x, y, t)}{\partial y^{2}}+\frac{d-1}{y} \frac{\partial C^{p}(x, y, t)}{\partial y}\right) \\
-\rho_{s} k C^{p}(x, y, t)\left(1-\theta^{p}(x, y, t)\right), & \\
& L_{1}<x<L_{1}+L_{2} . \quad 0 \leq y \leq R, \quad 0 \leq t<+\infty,
\end{aligned}
$$

- the interface equations for the concentration $C$ at the boundaries between zones, expressing continuity of concentration

$$
C\left(L_{1}^{-}, t\right)=C\left(L_{1}^{+}, t\right), \quad C\left(L_{1}+L_{2}^{-}, t\right)=C\left(L_{1}+L_{2}^{+}, t\right), \quad 0 \leq t<+\infty
$$

and of flux:

$$
\begin{gathered}
-D_{1} \frac{\partial C}{\partial x}\left(L_{1}^{-}, t\right)=-D_{2} \frac{\partial C}{\partial x}\left(L_{1}^{+}, t\right), \quad 0 \leq t<+\infty, \\
-D_{2} \frac{\partial C}{\partial x}\left(L_{1}+L_{2}^{-}, t\right)=-D_{3} \frac{\partial C}{\partial x}\left(L_{1}+L_{2}^{+}, t\right), \quad 0 \leq t<+\infty,
\end{gathered}
$$

- the reaction equation for the coverage $\theta$ in the second zone,

$$
\frac{\partial \theta^{p}(x, y, t)}{\partial t}=k C^{p}(x, y, t)\left(1-\theta^{p}(x, y, t)\right), \quad L_{1}<x<L_{1}+L_{2}, 0 \leq y \leq R, 0 \leq t<+\infty,
$$

- boundary conditions in $y$ : no diffusional limitation at the external surface of the catalyst support,

$$
C^{p}(x, R, t)=C(x, t), \quad L_{1}<x<L_{1}+L_{2}, \quad 0 \leq t<+\infty,
$$

- and symmetry at the centre.

$$
\frac{\partial C^{p}}{\partial y}(x, 0, t)=0, \quad L_{1}<x<L_{1}+L_{2}, \quad 0 \leq t<+\infty .
$$

- the boundary condition on $C$ at the reactor inlet (where $x=0$ ),

$$
-D_{1} \frac{\partial C}{\partial x}(0, t)=\frac{N_{p}}{A} I(t), \quad 0 \leq t<+\infty,
$$

- the boundary condition on $C$ at the reactor outlet (where $x=L_{1}+L_{2}+L_{3}$ )

$$
C\left(L_{1}+L_{2}+L_{3}, t\right)=0, \quad 0 \leq t<+\infty,
$$

- the initial conditions on $C$ and $C^{p}$ (empty reactor),

$$
C(x, 0)=C^{p}(x, y, 0)=0, \quad 0 \leq x \leq L_{1}+L_{2}+L_{3}, \quad 0 \leq y \leq R,
$$

- the initial coverage,

$$
\theta^{p}(x, y, 0)=0, \quad L_{1}<x<L_{1}+L_{2}, \quad 0<y<R .
$$



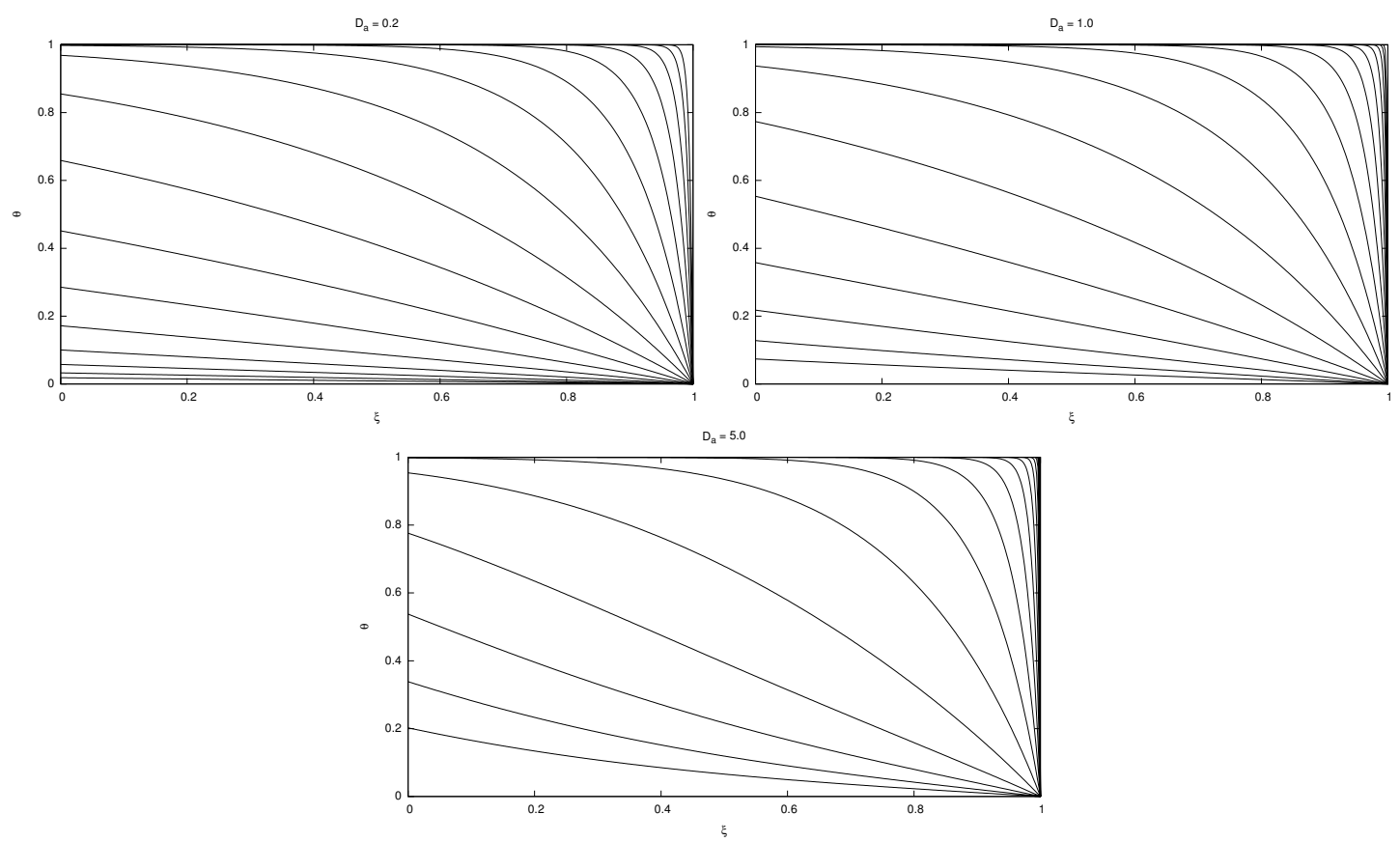

FiguRE 1. Single-zone, nonporous reactor: evolution of profiles corresponding to increasing dimensionless total amount pulsed in $\tilde{p}=\mathrm{Da} \cdot\left(10^{-1}, 10^{-3 / 4}, 10^{-2 / 4}, \ldots, 10^{3}\right)$ for three different $\mathrm{Da}=0.2,1.0,5.0$.

Mathematically, the treatment of this case mirrors that of the single-zone reactor, but the inert first and third zones must be taken into account, and the dependence on the internal variable $y$.

In this case the time integration is performed first at the pellet scale, writing $M^{p}(x, y)$ for the zeroth moment in time of $C^{p}(x, y, t)$ and obtaining from (3.8) that

$$
\theta_{+\infty}^{p}(x, y)=1-\left(1-\theta_{0}^{p}(x, y)\right) \exp \left(-k M^{p}(x, y)\right)
$$

and using this when integrating (3.4) similarly as before,

$$
\begin{gathered}
0=D_{p}\left(\frac{\partial^{2} M^{p}(x, y)}{\partial y^{2}}+\frac{d-1}{y} \frac{\partial M^{p}(x, y)}{\partial y}\right) \\
-\rho_{s}\left(1-\theta_{0}^{p}(x, y)\right)\left(1-\exp \left(-k M^{p}(x, y)\right)\right), \\
L_{1}<x<L_{1}+L_{2}, \quad 0 \leq y \leq R
\end{gathered}
$$

Integrating the other equations, at the reactor scale in terms of $M(x)$, is straightforward:

$$
0=\frac{D_{1} d^{2} M}{d x^{2}}=D_{2} \frac{d^{2} M}{d x^{2}}-\lambda\left(1-\epsilon_{2}\right) D_{p} \frac{d^{2} M^{p}}{d x^{2}}=D_{3} \frac{d^{2} M}{d x^{2}}
$$

in the three zones, with $M$ and its flux equivalent $-D(d M / d x)$ continuous at the interfaces and satisfying (2.13) at the inlet and (2.14) at the outlet. Again, the resulting system of partial differential equations determines uniquely the functions $M^{p}(x, y)$ and $M(x)$, and from the former the final coverage $\theta_{+\infty}^{p}(x, y)$ follows. Since in this system of partial differential equations $I(t)$ only occurs through its integral over time, the final coverage is again seen not to depend on any other property of the inlet pulse. Translating this mathematical result into physico-chemical language, it is proven that at any position the final concentration of catalytic sites is uniquely defined by the integral amount of gas substance concentration 
related to the given position. This holds because the process of gas-solid interaction is a single irreversible monomolecular adsorption. It its turn, the integral amount of gas molecules at this position is uniquely defined by the total amount of molecules that enter the chemical reactor. This holds due to the special properties of the TAP-reactor and its regime characteristics, i.e., the specific boundary conditions.

\section{Conclusions}

Whenever the partial differential equations allow to integrate the coverage in terms of the area under the concentration curve, differential equations are obtained that determine uniquely the final coverage as a function of the total amount of reactants admitted to the system; consequently the set of reachable final coverages can then be parametrised by a single value and is therefore vastly simplified compared to the general case, where it is typically infinite-dimensional.

Acknowledgements. Financial support from the Long Term Structural Methusalem Funding by the Flemish Government.

\section{References}

[1] K.P. De Jong. Synthesis of Solid Catalysts. Wiley-VCH, 2009.

[2] G. Ertl, H. Knözinger, J. Weitkamp. Preparation of Solid Catalysts. Wiley-VCH, 1999.

[3] J.T. Gleaves, J. Ebner, T. Kuechler. Temporal Analysis of Products (TAP) - a unique catalyst evaluation system with submillisecond time resolution. Catalysis Reviews - Science and Engineering 30 (1) (1988), 49-116.

[4] J.T. Gleaves, G. Yablonsky, X. Zheng, R. Fushimi, P.L. Mills. Temporal analysis of products (TAP) - Recent advances in technology for kinetic analysis of multi-component catalysts. Journal of Molecular Catalysis A - Chemical 315 (2, SI) (2010), 108-134.

[5] S.Y. Lee, R. Aris. The Distribution of Active Ingredients in Supported Catalysts Prepared by Impregnation. Catalysis Reviews - Science and Engineering 27 (2) (1985), 207-340.

[6] G.B. Marin, G.S. Yablonsky. Chemical Kinetics: Decoding Complexity. J. Wiley - VCB, 2011.

[7] M. Morbidelli, A. Gavrilidis, A. Varma. Catalyst Design: Optimal Distribution of Catalyst in Pellets, Reactors and Membranes. Cambridge University Press, 2001.

[8] E.E. Petersen, A.T. Bell. Catalyst Deactivation. CRC Press, 1987.

[9] P. Phanawadee, N. Pongboutr, G.S. Yablonsky, D. Constales, C. Jarungmanorom, W. Soikham, J. Limtrakul. Independence of Active Substance Profiles from the Pulse Response Experimental Procedure. AIChE Journal 59 (2013) 3574-3577.

[10] J. Regalbuto. Catalyst Preparation: Science and Engineering. Taylor and Francis/CRC Press. 2007. 